Fluorescence (TALIF) measurement of atomic hydrogen concentration in a coplanar surface dielectric barrier discharge

This content has been downloaded from IOPscience. Please scroll down to see the full text. 2016 Plasma Sources Sci. Technol. 25055015

(http://iopscience.iop.org/0963-0252/25/5/055015)

View the table of contents for this issue, or go to the journal homepage for more

Download details:

IP Address: 147.251.27.66

This content was downloaded on 29/08/2016 at 09:36

Please note that terms and conditions apply.

You may also be interested in:

Optical diagnostics of reactive species in atmospheric-pressure nonthermal plasma

Ryo Ono

Distribution of $\mathrm{H}$ atoms in a plasma reactor

$\mathrm{X} R$ Duan, $\mathrm{H}$ Lange and A Meyer-Plath

Nitric oxide kinetics in the afterglow of a diffuse plasma filament

D Burnette, A Montello, I V Adamovich et al.

Surface charge dynamics and $\mathrm{OH}$ and $\mathrm{H}$ number density distributions in near-surface nanosecond pulse discharges at a liquid / vapor interface

Caroline Winters, Vitaly Petrishchev, Zhiyao Yin et al.

Laser-induced resonance fluorescence as a diagnostic technique in non-thermal equilibrium plasmas

J Amorim, G Baravian and J Jolly

Absolute calibration ofatomic density in TALIF

K Niemi, V Schulz-von der Gathen and H F Döbele

Laser-induced fluorescence measurements

H F Döbele, T Mosbach, K Niemi et al. 


\title{
Fluorescence (TALIF) measurement of atomic hydrogen concentration in a coplanar surface dielectric barrier discharge
}

\author{
M Mrkvičková, J Ráhel', P Dvořák, D Trunec and T Morávek \\ Department of Physical Electronics, Faculty of Science, Masaryk University, Kotlářská 2, \\ Brno 611 37, Czech Republic \\ E-mail: martinamrkvickova@gmail.com
}

Received 15 January 2016, revised 27 May 2016

Accepted for publication 22 June 2016

Published 25 August 2016

\begin{abstract}
Spatially and temporally resolved measurements of atomic hydrogen concentration above the dielectric of coplanar barrier discharge are presented for atmospheric pressure in $2.2 \%$ $\mathrm{H}_{2}$ /Ar. The measurements were carried out in the afterglow phase by means of two-photon absorption laser-induced fluorescence (TALIF). The difficulties of employing the TALIF technique in close proximity to the dielectric surface wall were successfully addressed by taking measurements on a suitable convexly curved dielectric barrier, and by proper mathematical treatment of parasitic signals from laser-surface interactions. It was found that the maximum atomic hydrogen concentration is situated closest to the dielectric wall from which it gradually decays. The maximum absolute concentration was more than $10^{22} \mathrm{~m}^{-3}$. In the afterglow phase, the concentration of atomic hydrogen above the dielectric surface stays constant for a considerable time $(10 \mu \mathrm{s}-1 \mathrm{~ms})$, with longer times for areas situated farther from the dielectric surface. The existence of such a temporal plateau was explained by the presented 1D model: the recombination losses of atomic hydrogen farther from the dielectric surface are compensated by the diffusion of atomic hydrogen from regions close to the dielectric surface. The fact that a temporal plateau exists even closest to the dielectric surface suggests that the dielectric surface acts as a source of atomic hydrogen in the afterglow phase.
\end{abstract}

Keywords: laser-induced fluorescence, TALIF, atomic hydrogen, $\mathrm{H}$, dielectric barrier discharge, surface discharge

(Some figures may appear in colour only in the online journal)

\section{Introduction}

Atomic hydrogen is a highly reactive species that plays an important role in the plasma treatment of solid surfaces and plasma chemistry. As a strong reducing and etching agent, atomic hydrogen is used in numerous low-pressure plasma applications, e.g. plasma etching [1], the passivation of defects in silicon industry [2], the removal of weakly bonded compounds during the deposition of hard and resistant thin films [3] etc. Recently, some promising results for the surface hydrogenation of diamond nanoparticles with atmospheric-pressure hydrogen plasma were accomplished in our laboratories [4], using coplanar dielectric barrier discharge (DBD). However, any wider use of atmospheric-pressure hydrogen plasma treatment raises serious safety concerns on account of high hydrogen flammability when hydrogen is mixed even with a small percentage of oxygen or air [5]. A simple, yet effective approach to mitigate these concerns is to dilute hydrogen with an inert gas, typically argon, to widen its flammability envelope [6]. The drawback of this approach is lower atomic hydrogen generation due to the reduced concentration of $\mathrm{H}_{2}$. Fortunately, the presence of Ar atoms introduces several novel 
reaction channels for $\mathrm{H}$ atom creation, which may to some extent compensate for the effect of $\mathrm{H}_{2}$ dilution. Two main atomic hydrogen production channels (i.e. electron impact dissociation: e $+\mathrm{H}_{2} \rightarrow \mathrm{H}+\mathrm{H}$, and ion-electron dissociative recombination $\mathrm{H}_{3}^{+}+\mathrm{e}^{-} \rightarrow \mathrm{H}_{2}+\mathrm{H}$ ) are extended by dissociation by metastables (i.e. $\mathrm{Ar}^{*}+\mathrm{H}_{2} \rightarrow \mathrm{Ar}+2 \mathrm{H}$ ), and by $\mathrm{ArH}^{+}$ ion-electron dissociative recombination (i.e. $\mathrm{ArH}^{+}+\mathrm{e}^{-} \rightarrow$ $\mathrm{Ar}+\mathrm{H})$ [7]. Furthermore, in a confined discharge space of $\mathrm{DBD}$, argon heterogeneous reactions on the dielectric walls ought to be considered as well. This includes the surface dissociative recombination of $\mathrm{ArH}^{+}$ions and various Ar-mediated Eley-Rideal mechanism reactions with adsorbed atomic and molecular hydrogen [8]. It should be noted here that the actual contribution of heterogeneous reactions is generally only estimated, and most numerical models do not take into account e.g. material-specific interactions [7]. Such deficiency is quite bewildering when we realize that the most common motivation for generating atomic hydrogen is to use it just for heterogeneous reactions on solid surfaces.

In the presented work we report on our experimental assessment of the ground-state atomic hydrogen concentration above the surface of coplanar DBD, operated at atmospheric pressure in a mixture of argon and hydrogen. Knowledge of the spatio-temporal evolution of atomic hydrogen provides an important insight into the ongoing chemical processes. The electric field distribution of coplanar DBD allows discharge microfilaments to propagate only in the thin (a few tenths of a $\mathrm{mm}$ ) layer along the dielectric surface, which results in a strong spatial gradient of created species. Owing to this, the experimental technique of choice must offer a high degree of spatial resolution. In this respect, a suitable method is twophoton absorption laser-induced fluorescence (TALIF), which has become a powerful tool for the detection of reactive species in plasma [9-14]. When TALIF is used for the detection of free hydrogen atoms, these atoms are usually excited by the simultaneous absorption of two laser photons with wavelength of $205.08 \mathrm{~nm}$ from the ground state to the state $n=3$ $[15,16]$. This leads to the generation of fluorescent $\mathrm{H} \alpha$ radiation with an intensity proportional to the atomic hydrogen concentration. The necessity of absorbing two-photons makes the emission of the $\mathrm{H} \alpha$ line highly localized only to areas in the laser beam center, which allows the acquisition of detailed $\mathrm{H}$ atom concentration maps.

The difficulty in employing TALIF measurement for probing a few tenths of a millimeter above the surface of coplanar DBD lies in our inability to completely prevent any contact of the laser beam with the dielectric. This leads not only to the detection of a redundant signal originating in the fluorescence of the dielectric material, but also to a laser-induced discharge breakdown [18]. Therefore, care must be taken to make the TALIF method non-invasive. The issue of artificial breakdown can be solved by synchronizing laser excitation with the DBD voltage cycle, so the laser shots are taken at the lowest internal field, insufficient to initiate artificial breakdown $[19,20]$. Another approach presented in this work is to take measurements in a repetitive afterglow regime, created by a modulated power feed pattern. Besides

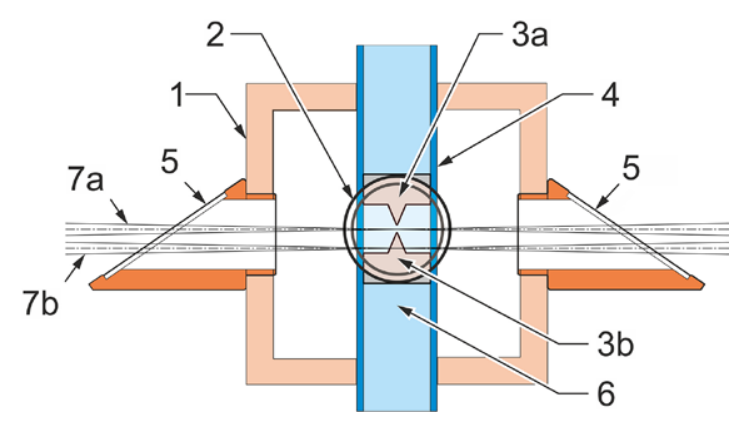

Figure 1. The dielectric barrier discharge reactor. 1-chamber, 2-quartz window for fluorescence observation, 3a-upper electrode, 3b-bottom electrode, 4-quartz tube, 5-quartz windows tilted to the Brewster angle, 6-insulation oil, 7-laser beam in position for the first (7a) and the second (7b) set of measurements.

simplified chemical kinetics of the afterglow phase, it has allowed us to monitor the development of atomic hydrogen over a longer time range, thus enabling a better validation of our numerical model. The issue of solid material fluorescence has been successfully addressed by adopting a suitable geometry of the DBD surface, which minimizes laser beam surface impingement. As we will report in this work, the geometry adaptation enabled us to monitor the afterglow $\mathrm{H}$-atom evolution of a single, spatially stabilized coplanar DBD microfilament.

\section{Experiment}

\subsection{Discharge configuration}

The schematic of the investigated surface coplanar dielectric barrier discharge is shown in figure 1. A spatially stabilized single discharge microfilament was ignited on the outer surface of a cylindrical quartz tube (HSQ 300, Heraeus) by a pair of brass triangular electrodes, situated in the tube interior and oriented in parallel to the tube central axis. The tube has an inner diameter of $25 \mathrm{~mm}$ and a wall thickness of $1 \mathrm{~mm}$. A relatively small radius of curvature of the quartz tube was adopted intentionally, to effectively divert the dielectric surface from the line of the laser beam, and thus to minimize the parasitic material fluorescence of out-of-focus laser beams impinging on the surface. A quartz dielectric barrier was used for three main reasons: (1) its high thermal shock resistance; (2) its low UV absorption leading to the low intensity of the fluorescence of the dielectric; (3) its smooth surface which minimizes laser radiation scattering to the detector. The latter allowed focusing the laser beam into the closest part of the dielectric surface.

To provide necessary HV electrode insulation and active cooling of the discharge site, insulation oil (Dow Corning 561 silicone transformer liquid) was circulated through the quartz tube. The distance between the electrode tips was set to $4 \mathrm{~mm}$. The tube was housed inside a closed plastic chamber to allow working gas control. In the presented experiment, a mixture of $450 \mathrm{sccm}$ of argon and $10 \mathrm{sccm}$ of hydrogen was continuously flown through the reactor; the whole experiment was carried out at atmospheric pressure. 


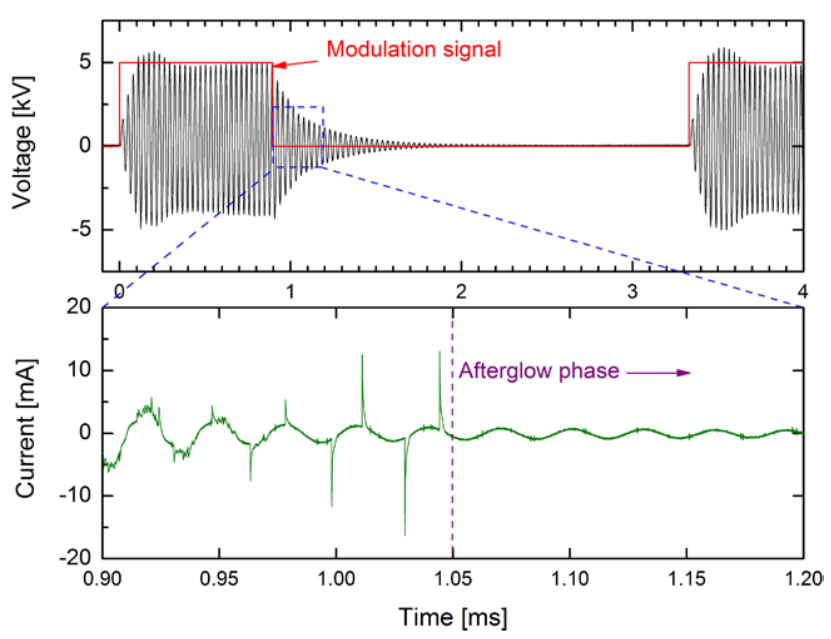

Figure 2. Voltage and current waveforms of the modulated coplanar surface DBD.

In-house-built HV power supply was used to power the electrodes by a $33.33 \mathrm{kHz} \mathrm{HV}$ driving voltage, modulated by a $300 \mathrm{~Hz}$ modulation signal with a duty cycle of $30 \%$. Specifically, a train of 30 discharge cycles with a single-cycle period of $30 \mu$ s was followed by an off-time (an afterglow phase) and the whole process was repeated at a $300 \mathrm{~Hz}$ repetition rate (figure 2). Upon entering the off-time of the modulation signal, the amplitude of the applied voltage oscillations started to decrease. To our benefit, the gradual decrease of the applied voltage resulted in an improved temporal stability of the microdischarge, as can be seen on the discharge current waveform in figure 2. Modulation of the HV power supply was done by the external arbitrary waveform generator (Rigol DG4162) operating in the repetitive $\mathrm{N}$ cycle burst mode. This waveform generator was simultaneously used for generating the laser shot synchronization signal. Digital oscilloscope DSO-S204A (Keysight) was used to monitor the discharge current, the applied voltage and the time position of the laser shot. An HV probe P6015A (Tektronix) and current monitor Model 2877 (Pearson) were used.

\subsection{Diagnostics setup}

A laser beam for TALIF measurement was generated by a three-component laser system, shown in figure 3. Pumping Nd:YAG laser (Spectra-Physics, Quanta-Ray PRO-270-30) produced radiation with a wavelength of $1064 \mathrm{~nm}$, doubled to $532 \mathrm{~nm}$ utilizing a KDP crystal. The $532 \mathrm{~nm}$ radiation was used for pumping a dye laser (Sirah, PrecisionScan PRSCD-24-EG) with rhodamine 101/B generating a wavelength of $615 \mathrm{~nm}$, tripled to $205 \mathrm{~nm}$ in a frequency conversion unit. The output laser beam was focused by a spherical lens with a focal length of $35 \mathrm{~cm}$ and passed through the discharge reactor in the vicinity of the dielectric. Two sets of positions of the laser radiation were used for the measurement: for the first set, the vertical position of the focused laser radiation was located in the middle of the gap between the electrodes and the perpendicular distance of the laser beam from the surface of the dielectric was varied. The vertical position of the laser

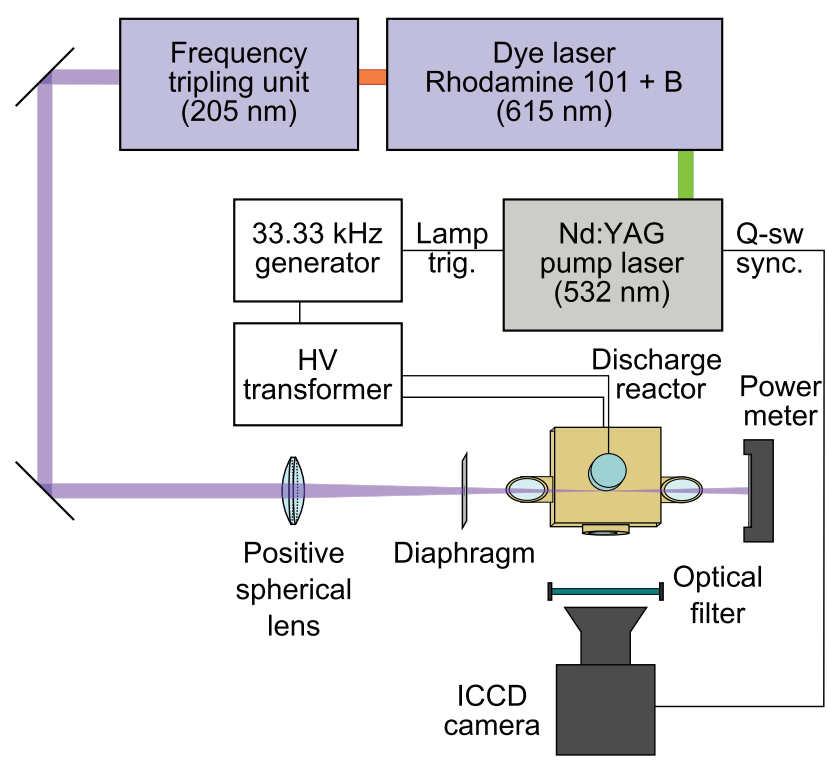

Figure 3. Experimental setup.

radiation in the second set of measurements was located in front of the bottom electrode; see figure 1. The areas used for the collection of fluorescence radiation in the two sets of measurements are depicted in figure 4. At the measurement point the radius of the laser beam was below $20 \mu \mathrm{m}$, which defined our spatial resolution of the measurement in the direction perpendicular to the dielectric surface. For improving the shape of the beam and for limiting its contact with the dielectric, an iris diaphragm was used. In order to minimize the reflections of the laser beam, the reactor windows were oriented at the Brewster angle. In order to perform timeresolved measurement, the pumping laser was synchronized with an HV power supply.

The fluorescence radiation was detected by an ICCD camera (Princeton Instruments, PI-MAX 1024RB-2-FG-43), which was synchronized with the pumping laser. To separate the measured signal from the plasma emission and laser scattering, an interference filter for the $\mathrm{H} \alpha$ line $656.3 \mathrm{~nm}$ was used.

\section{TALIF method}

\subsection{Measurement procedure}

The TALIF of hydrogen atoms is based on the excitation of the atoms from their ground state $1 \mathrm{~s}{ }^{2} \mathrm{~S}_{1 / 2}$ to the state $3 \mathrm{~d}^{2} \mathrm{D}_{3 / 2}, 5 / 2$ via the simultaneous absorption of two photons of laser radiation with a wavelength of $205.08 \mathrm{~nm}[15,16]$. Two-photon absorption enables one to overcome problems with the generation and manipulation of VUV radiation that would arise if standard single-photon laser-induced fluorescence (LIF) was used. The consequent radiative deexcitation of excited hydrogen atoms to $2 \mathrm{p}^{2} \mathrm{P}_{1 / 2,3 / 2}$ state produces fluorescence at $656.3 \mathrm{~nm}(\mathrm{H} \alpha)$, which is detected and its intensity is used for the calculation of the concentration of the hydrogen atoms in the ground state. The method can be calibrated by the TALIF measurement of krypton with known concentration. 


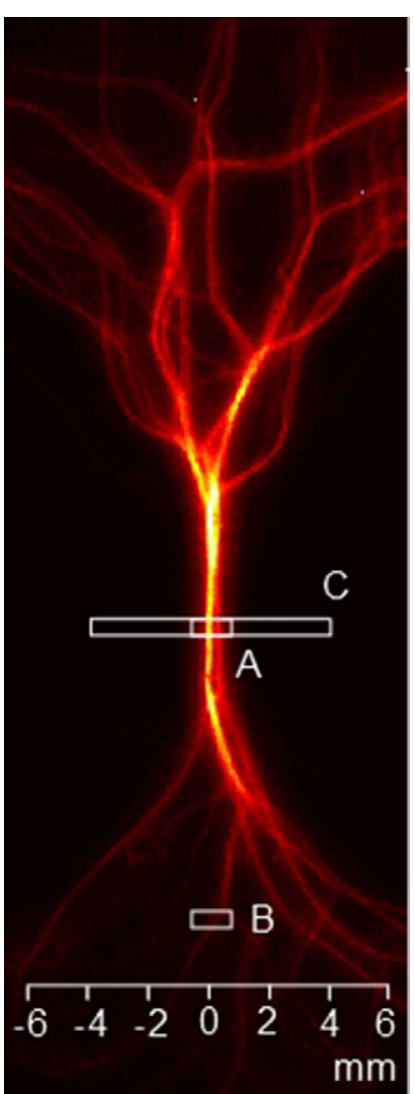

Figure 4. Discharge image with marked measurement positions. Rectangles A and B represent the measurement areas for the first and second measurement sets, respectively. Rectangle $\mathrm{C}$ represents the area used for spatially resolved measurements in the tangential direction, which are shown in figures 9 and 10 .

The measured signal was spectrally, spatially and temporally integrated. For each laser pulse, the ICCD camera was exposed for $50 \mathrm{~ns}$. This exposure time safely covered the whole laser pulse and the fluorescence decay time. After measuring the shape of the absorption line of hydrogen at given conditions, the other part of the experiment could be performed quickly with a laser tuned only to the center of the absorption line and the results were subsequently recalculated to spectral integrals. The measured signal was typically accumulated during 500 laser pulses and averaged over ten frames.

\subsection{Saturation}

Laser pulses with energies in the range of 50-60 $\mu \mathrm{J}$ were used for the TALIF of hydrogen atoms and energies of $10-20 \mu \mathrm{J}$ were used for the TALIF of krypton that was used for the calibration of the method. In these energy ranges, saturation effects emerged, which were demonstrated by the fact that the $\log -\log$ dependencies of the fluorescence signal on the energy of the laser pulses had slopes of approximately 1.7 and 1.3 for the TALIF of hydrogen and krypton, respectively. This slope would reach a value of 2 in an unsaturated case. It was not possible to decrease the laser energy enough to avoid saturation either for the hydrogen atoms or for krypton, due to the poor signal-to-noise ratio for low laser energies. Therefore, the saturation effects were taken into account, using the formula

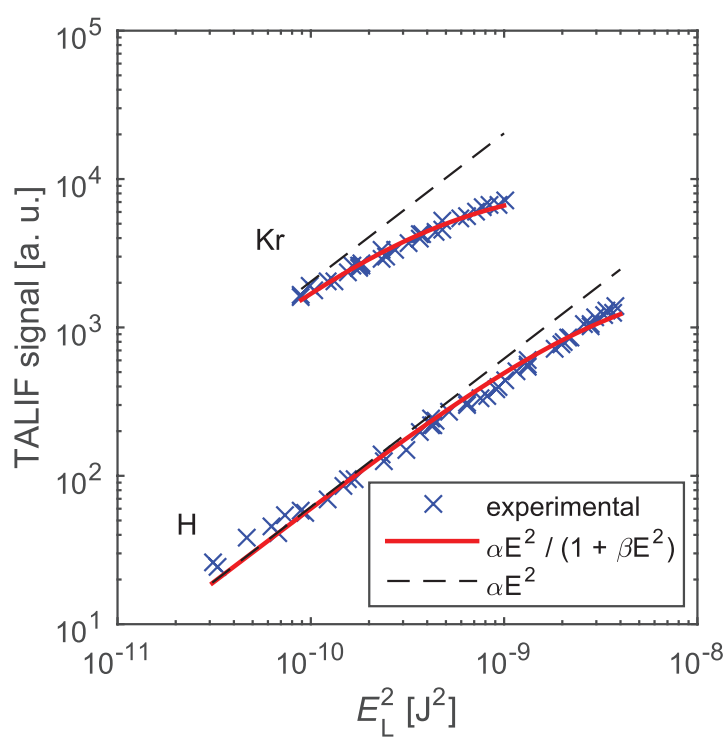

Figure 5. Fluorescence signal as a function of the energy of the laser pulses. The measurement data are fitted by equation (1). The dashed line shows the theoretical signal devoid of saturation effects.

$$
F=\frac{\alpha E_{\mathrm{L}}^{2}}{1+\beta E_{\mathrm{L}}^{2}},
$$

in analogy to the formula $F=\alpha E_{\mathrm{L}} /\left(1+\beta E_{\mathrm{L}}\right)$ that was derived for single-photon LIF [19]. $F$ is the measured fluorescence signal, $E_{\mathrm{L}}^{2}$ is the mean value of the squared laser-pulse energies, $\alpha E_{\mathrm{L}}^{2}$ would be the hypothetical unsaturated signal and the coefficient $\beta$ describes the saturation effects. When the coefficient $\beta$ is found, the measured fluorescence signal can be multiplied by $\left(1+\beta E_{\mathrm{L}}^{2}\right)$, which serves as the correction of saturation effects. The measured dependence of the fluorescence signal on the energy of the laser pulses is shown in figure 5, both for atomic hydrogen and for krypton. The formula (1) was fitted to these dependencies and the obtained coefficients $\beta$ were thereafter used for the correction of saturation in equation (2). Typical values of the correction are shown together with typical values of the laser-pulse energies in table 1 .

Finally, the effect of hydrogen atom production by the dissociation of hydrogen molecules in an intense laser beam was examined. Since there was no TALIF signal of atomic hydrogen when the discharge was not running, it was verified that this parasitic production of hydrogen atoms had no significant effect on the presented measurements.

\subsection{Laser-surface interaction}

Although the dielectric was made of quartz with a weak absorption of the used UV radiation and the interference filter in the camera was utilized, some amount of scattered laser light or the fluorescence of quartz was still detected when the laser beam touched the dielectric. This parasitic signal depended linearly on the energy of the laser pulse. Therefore, the following procedure was performed to separate the hydrogen fluorescence signal from other redundant signals. Besides a 
Table 1. Parameters used for the evaluation of TALIF measurements.

\begin{tabular}{lll}
\hline Parameter & $\mathrm{H}$ & $\mathrm{Kr}$ \\
\hline$\lambda$ & $205.08 \mathrm{~nm}$ & $204.13 \mathrm{~nm}$ \\
$q_{\mathrm{Ar}}$ & $3.93 \times 10^{-16} \mathrm{~m}^{3} \mathrm{~s}^{-1}[16]$ & $1.29 \times 10^{-16} \mathrm{~m}^{3} \mathrm{~s}^{-1}[16]$ \\
$q_{\mathrm{H}_{2}}$ & $20.4 \times 10^{-16} \mathrm{~m}^{3} \mathrm{~s}^{-1}[16]$ & \\
$q_{\mathrm{Kr}}$ & & $1.46 \times 10^{-16} \mathrm{~m}^{3} \mathrm{~s}^{-1}[16]$ \\
$\tau_{\mathrm{rad}}$ & $17.6 \mathrm{~ns}[16]$ & $34.1 \mathrm{~ns}[16]$ \\
$E_{\mathrm{L}}$ & $50-60 \mu \mathrm{J}$ & $10-20 \mu \mathrm{J}$ \\
$1+\beta E_{\mathrm{L}}^{2}$ & $1.6-1.9$ & $1.2-1.8$ \\
$T$ & $80 \%$ & $82 \%$ \\
$C$ & $5.2 \%$ & $1.8 \%$ \\
\hline$\sigma_{\mathrm{Kr}}^{\mathrm{TA}} / \sigma_{\mathrm{H}}^{\mathrm{TA}}$ & & $0.62[16]$ \\
$A_{\mathrm{Kr}} / A_{\mathrm{H}}$ & & $0.614[21]$ \\
\hline
\end{tabular}

Note: $\lambda$ is the laser wavelength, $q_{\mathrm{Ar}}, q_{\mathrm{H}_{2}}$ and $q_{\mathrm{Kr}}$ are the rate coefficients for the quenching of excited species at room temperature by binary collisions with $\mathrm{Ar}, \mathrm{H}_{2}$ and $\mathrm{Kr}$, respectively, $\tau_{\mathrm{rad}}$ is the radiative lifetime of excited species and $E_{\mathrm{L}}$ is the typical range of the energy of the laser pulses. The remaining quantities are described in section 3 .

common dark frame measured without the presence of any laser radiation, each measurement was repeated with the laser detuned some $20 \mathrm{pm}$ from the center of the hydrogen absorption line, i.e. at a wavelength that cannot excite hydrogen atoms but generates practically the same intensity of the fluorescence of the dielectric. The laser-pulse energy of both measurements was kept at similar levels. The pure fluorescence signal of hydrogen $S$ was calculated as

$S=\left[S_{\text {meas }}-S_{\text {dark }}-\left(S_{\text {detuned }}-S_{\text {dark }}\right) \cdot \frac{E_{\mathrm{L}, \text { meas }}}{E_{\mathrm{L}, \text { detuned }}}\right] \cdot\left(1+\beta E_{\mathrm{L}, \text { meas }}^{2}\right)$,

where $S_{\text {meas }}$ is the signal measured with the laser wavelength tuned to the center of the absorption line; $E_{\mathrm{L} \text {,meas }}$ is the mean value of the laser-pulse energies during this main measurement; $S_{\text {detuned }}$ is the signal measured with the laser detuned some $20 \mathrm{pm}$ from the center of the absorption line; $E_{\mathrm{L}, \text { detuned }}$ is the mean value of the energies of the detuned laser pulses; $S_{\text {dark }}$ is the dark signal measured without any laser radiation; and expression $\left(1+\beta E_{\mathrm{L} \text {,meas }}^{2}\right)$ compensates for the saturation effects. The subtracted parasitic signal $\left(S_{\text {detuned }}-S_{\text {dark }}\right)$ was well below $20 \%$ of the signal $\left(S_{\text {meas }}-S_{\text {dark }}\right)$.

\subsection{Absolute concentration values}

To determine the absolute densities of the hydrogen atoms, calibration measurement of the TALIF of krypton was performed. A mixture of $\mathrm{Ar}+3 \% \mathrm{Kr}$ was injected into the reactor. The krypton atoms were excited from the ground state $4 \mathrm{p}^{6}{ }^{1} \mathrm{~S}_{0}$ to the $5 \mathrm{p}$ ' $[3 / 2]_{2}$ state by the two-photon absorption of $204.13 \mathrm{~nm}$ laser radiation. Subsequent fluorescence radiation was observed on wavelength $826.3 \mathrm{~nm}$ during the deexcitation to $5 s^{\prime}[1 / 2]_{1}$ state. The absolute concentrations of hydrogen atoms $N_{\mathrm{H}}$ were calculated by the formula [16]

$$
N_{\mathrm{H}}=N_{\mathrm{Kr}} \frac{S_{\mathrm{H}} E_{\mathrm{L}, \mathrm{Kr}}^{2}}{S_{\mathrm{Kr}} E_{\mathrm{L}, \mathrm{H}}^{2}}\left(\frac{\nu_{\mathrm{H}}}{\nu_{\mathrm{Kr}}}\right)^{2} \frac{\sigma_{\mathrm{Kr}}^{\mathrm{TA}}}{\sigma_{\mathrm{H}}^{\mathrm{TA}}} \frac{A_{\mathrm{Kr}} \tau_{\mathrm{Kr}}}{A_{\mathrm{H}} \tau_{\mathrm{H}}} \frac{T_{\mathrm{Kr}}}{T_{\mathrm{H}}} \frac{C_{\mathrm{Kr}}}{C_{\mathrm{H}}},
$$

where $S$ represents the temporally, spatially and spectrally integrated fluorescence signals with the correction of saturation effects; $E_{\mathrm{L}}^{2}$ is the mean value of the squared laser-pulse energies; $\nu$ is the frequency of the laser photons; $\sigma^{\text {TA }}$ is the cross section for two-photon absorption; $A$ is the Einstein coefficient of spontaneous emission from the excited state; $\tau$ is the lifetime of the excited state (fluorescence decay time); $T$ is the transmission of the interference filter; and $C$ is the quantum efficiency of the ICCD camera for the wavelength of the fluorescence. Indices ' $\mathrm{H}$ ' and ' $\mathrm{Kr}$ ' differentiate between quantities related to atomic hydrogen and krypton, respectively. The ratio of the two-photon absorption cross sections for atomic hydrogen and krypton was published in $[15,16]$.

Since the lifetime of excited hydrogen and krypton atoms was approximately $0.1 \mathrm{~ns}$ and $0.3 \mathrm{~ns}$, respectively, it was not possible to determine the lifetimes experimentally. Therefore, they were calculated by means of known quenching coefficients that were taken from [16, 17]. The values of the quenching rate coefficients are summarized in table 1 together with other quantities used for the evaluation of the TALIF measurements in this work. The effect of quenching by threebody collisions was neglected, which is in agreement with the findings described in [22]. However, the temperature dependence of the quenching rates should be taken into account. The values of quenching rate constants depend on the mean speed of the colliding particles and the concentration of collisional partners is a function of the gas temperature as well. The mutual effect of these two factors predicts that the quenching rate is proportional to $T_{\mathrm{g}}^{-0.5}$, where $T_{\mathrm{g}}$ is the gas temperature. According to spectral measurements of the rotational temperature, the value $500 \mathrm{~K}$ was used as the gas temperature in the coplanar discharge, whereas the $\mathrm{Ar}-\mathrm{Kr}$ mixture used for the calibration measurements was assumed to have room temperature. The eventual cooling of the gas in the afterglow phase of the DBD was not analyzed. Consequently, it is possible that the lifetime of the excited hydrogen $\left(\tau_{\mathrm{H}}\right)$ decreased slightly with time due to the gas cooling in the afterglow phase. Therefore, the real concentration of hydrogen atoms in the late phases of the afterglow or in areas with lower gas temperature might have been a little higher than the values presented in this work. As a result, it is possible that the real duration of the plateau shown in section 4 was in reality slightly longer than is presented in the forthcoming section. Nevertheless, even in the case of complete gas cooling to room temperature during the first $2 \mathrm{~ms}$ of the afterglow, the error of the calculated concentration values would be less than $30 \%$.

\section{Results and discussion}

\subsection{Atomic hydrogen concentration}

The concentration of atomic hydrogen in various positions is shown in figures 6 and 7. Immediately after the end of the discharge pulse, the concentration values varied in the range of $10^{21}-2 \cdot 10^{22} \mathrm{~m}^{-3}$. The highest values were found in the vicinity of the dielectric surface, where the hydrogen dissociation degree defined as $[\mathrm{H}] /\left([\mathrm{H}]+2\left[\mathrm{H}_{2}\right]\right)$ reached approx. $3 \%$. 


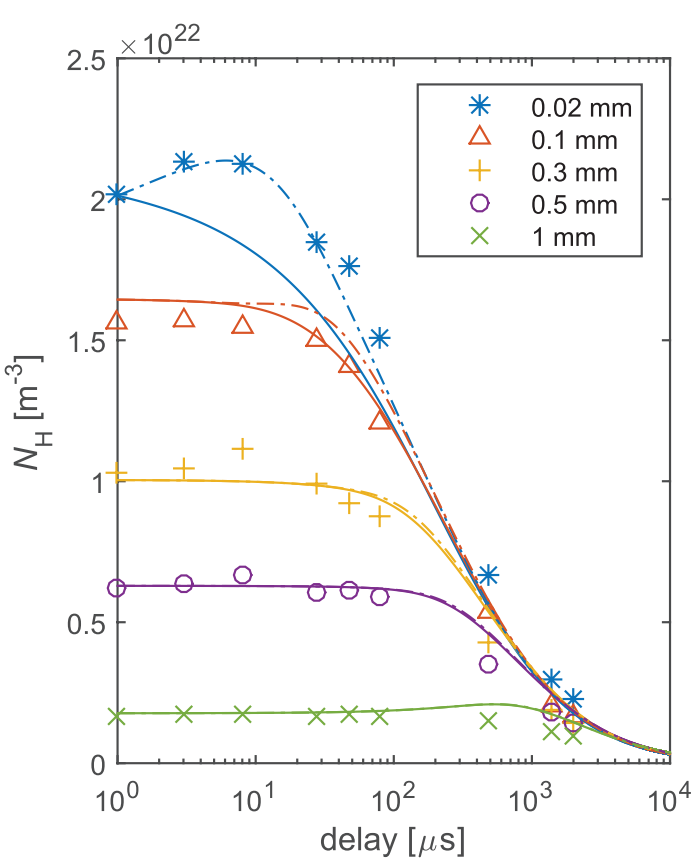

Figure 6. Temporal evolution of atomic hydrogen concentration in the afterglow for the first set of positions (between electrodes) for various distances from the dielectric surface. The lines represent the results of the diffusion-recombination model with (dash-and-dot) or without (solid lines) the source term on the surface.

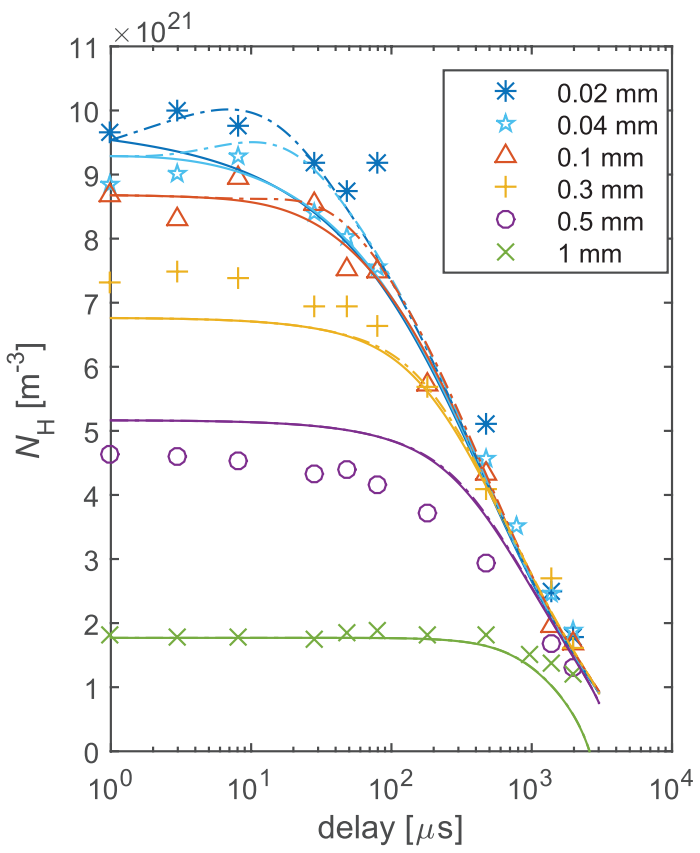

Figure 7. Temporal evolution of atomic hydrogen concentration in the afterglow for the second set of positions (at the electrode) for various distances from the dielectric surface. The lines represent the results of the diffusion-recombination model with (dash-and-dot) or without (solid lines) the source term on the surface.

This concentration value can be compared with the atomic hydrogen concentration found in other plasma sources. In an atmospheric pressure volume DBD, concentration over $10^{21} \mathrm{~m}^{-3}$ was measured by the TALIF method [22]. In lowpressure capacitively coupled discharges the atomic hydrogen

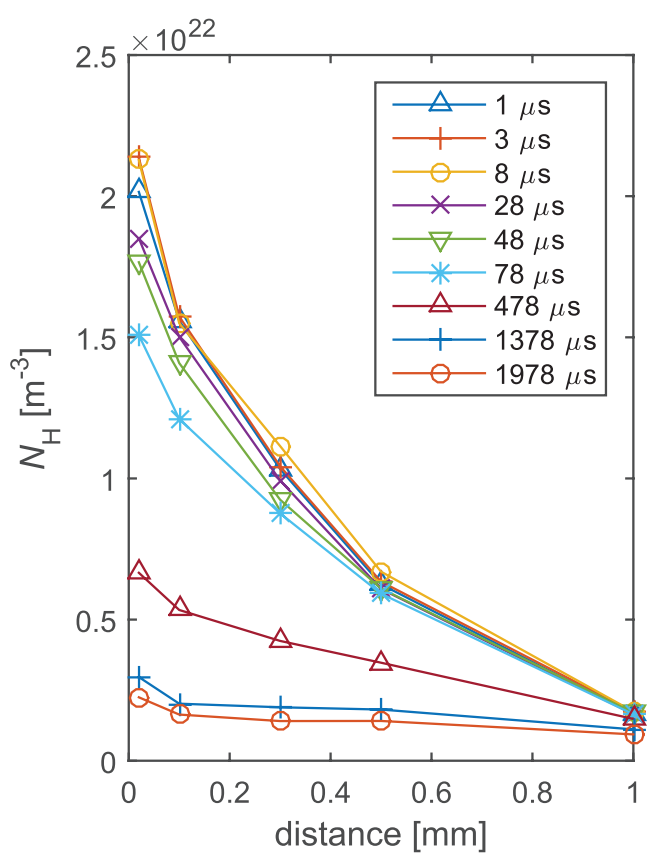

Figure 8. Spatial distribution of atomic hydrogen in the plane of symmetry of the reactor; the position shows the distance from the dielectric surface (in front of the inter-electrode gap). Shown for various delay times after the end of the discharge pulse.

concentration was found to be in the order of $10^{20} \mathrm{~m}^{-3}$ by means of TALIF $[21,23]$ and in the range of orders from $10^{20}$ to $10^{22} \mathrm{~m}^{-3}$ by means of a method based on the comparison of atomic and molecular line intensities [24]. The same method was used in hydrogen capillary DC-arc discharge and in MW discharge. In both these discharge types, the atomic hydrogen concentration was found to be in the order of $10^{20} \mathrm{~m}^{-3}$ [24]. Finally, in the expansion region of an $\mathrm{Ar}-\mathrm{H}_{2}$ arc the concentration over $10^{20} \mathrm{~m}^{-3}$ was determined by the TALIF method [15]. It follows from this comparison that the concentration of atomic hydrogen in the surface coplanar DBD is relatively high, which can be most probably attributed to a high power density in its thin active discharge layer.

As described above, the concentration of free hydrogen atoms was measured in two sets of positions. The first set of measurements was located on the horizontal plane of symmetry of the discharge, i.e. between electrodes. The measurements were repeated for various distances of the focused laser beam from the surface of the dielectric. The atomic hydrogen concentration was averaged over approx. $1 \mathrm{~mm}$ of the laser beam path. This measurement area is depicted in figure 4 by rectangle $\mathrm{A}$. The temporal evolution of atomic hydrogen after the end of the discharge pulse in this set of positions is shown in figure 6 for various distances from the surface of the dielectric. It can be seen that after the end of the discharge pulse, atomic hydrogen concentration remained constant for 10-1000 $\mu$ s and only after this extended time did it start to fall. The duration of the concentration plateau depended on the distance from the surface of the dielectric: it terminated after tens of microseconds in the closest vicinity of the surface but it lasted for approximately $1 \mathrm{~ms}$ in the distance of $1 \mathrm{~mm}$ from the surface. Figure 7 depicts an analogous set of 


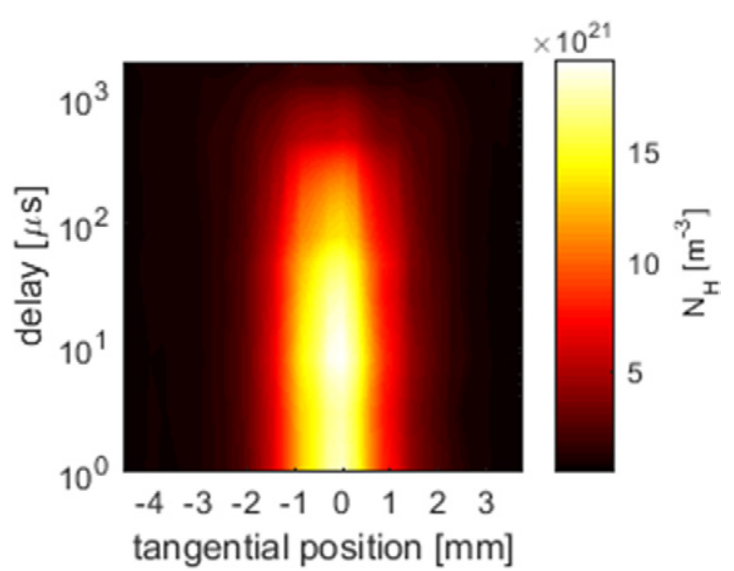

Figure 9. Spatio-temporal development of atomic hydrogen concentration in the distance $0.1 \mathrm{~mm}$ from the dielectric surface.

measurements realized in front of the bottom electrode (rectangle B in figure 4). The temporal and spatial development of atomic hydrogen at the electrode is similar to the situation in front of the gap, but the concentration values are smaller in front of the electrode, for the filaments are not pinned to a stationary position here as they were at the inter-electrode gap. Atomic hydrogen concentration again decreases when distance from the surface is increased and the temporal evolution again reveals a plateau with slightly longer duration than that in front of the inter-electrode gap. Our explanation for the existence of the plateau will be presented in section 4.2.

The data in figures 6 and 7 can be inverted in order to show the spatial distribution of the atomic hydrogen concentration. The atomic hydrogen spatial distribution in front of the interelectrode gap is shown in figure 8; the situation in front of the bottom electrode is not shown since it is very similar to the situation in front of the gap. Shortly after the end of the discharge pulse, the spatial profile of atomic hydrogen concentration reveals a nearly exponential decrease with a fall to one half of the maximal concentration at the $0.3 \mathrm{~mm}$ distance from the dielectric surface. This finding is in agreement with the optical measurement of the thickness of the active surface coplanar DBD layer reported in [25]. When the time spent in the afterglow phase was increased, atomic hydrogen concentration decreased and its profile was flattened by the diffusion flow and recombination losses in agreement with our model described in section 4.2.

Since the ICCD camera directly measured the image of the fluorescence, we could quite straightforwardly analyze the atomic hydrogen distribution along the laser beam, i.e. in the tangential direction to the discharge axis, as depicted by rectangle $\mathrm{C}$ in figure 4. A typical example of the atomic hydrogen concentration spatio-temporal map is shown in figure 9, where spatio-temporal development in the distance $0.1 \mathrm{~mm}$ from the dielectric surface is depicted. The shape of the spatiotemporal dependencies in other measurement lines in front of the inter-electrode gap was similar, but the maximum concentration decreased with the distance from the dielectric surface increased. Figure 9 demonstrates that a plateau is also presented alongside the discharge axis with practically the same duration. The width of the atomic hydrogen-rich region can

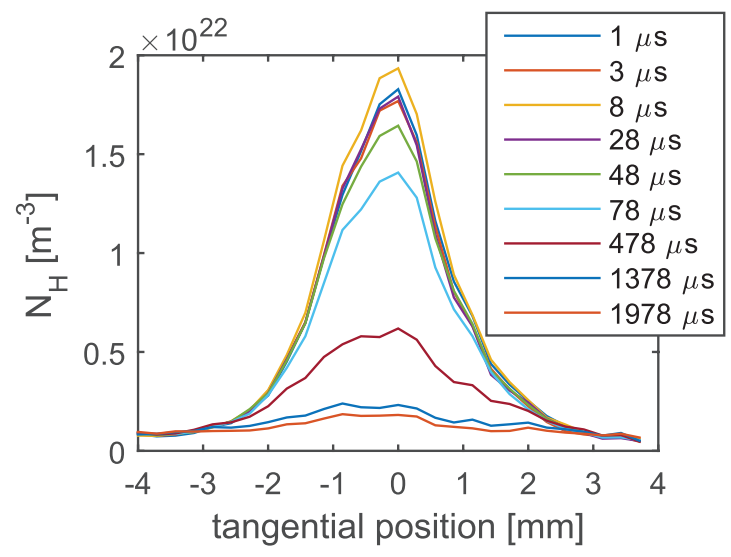

Figure 10. The spatial distribution of atomic hydrogen concentration in the direction of the laser beam in the distance $0.1 \mathrm{~mm}$ from the dielectric surface for various delay times from the end of the discharge pulse.

also be seen in figure 10, which shows cuts of figure 9 for decay times of $1 \mu$ s to approx. 2 ms. The FWHM rises from an initial $2 \mathrm{~mm}$ to approx. $3.5 \mathrm{~mm}$ for a delay time of $2 \mathrm{~ms}$. A different situation was found in front of the electrode, where the spatial profile of atomic hydrogen was flat due to the random position of the discharge filaments. This flat concentration profile and the resulting weak diffusion parallel to the surface can explain the fact that the plateau persisted slightly longer in front of the electrode than in front of the inter-electrode gap.

\subsection{Model of atomic hydrogen diffusion and recombination}

The development of $\mathrm{H}$ atom concentration $n(x, t)$ in front of the dielectric surface was described by the following onedimensional (1D) equation:

$$
\frac{\partial n(x, t)}{\partial t}=D \frac{\partial n(x, t)}{\partial x}-k_{\mathrm{r}}[n(x, t)]^{2},
$$

where $x$ is the distance above the dielectric, $D$ is the diffusion coefficient for hydrogen atoms in $\operatorname{argon}$ and $k_{\mathrm{r}}$ is the recombination coefficient. $k_{\mathrm{r}}$ is the sum of recombination coefficients $k_{\mathrm{r} 1} n_{\mathrm{Ar}}$ and $k_{\mathrm{r} 2} n_{\mathrm{H}_{2}}$ for two possible recombination channels

$$
\mathrm{H}+\mathrm{H}+\mathrm{Ar} \rightarrow \mathrm{H}_{2}+\mathrm{Ar} \quad k_{\mathrm{r} 1}=1.8 \times 10^{-30} / T_{\mathrm{g}}
$$

and

$$
\mathrm{H}+\mathrm{H}+\mathrm{H}_{2} \rightarrow \mathrm{H}_{2}+\mathrm{H}_{2} \quad k_{\mathrm{r} 2}=2.7 \times 10^{-31} T_{\mathrm{g}}^{-0.6},
$$

where the recombination coefficient units are $\mathrm{cm}^{6}$ molecule $\mathrm{e}^{-2} \mathrm{~s}^{-1}$ and $T_{\mathrm{g}}$ is the gas temperature. The temperature of $\mathrm{H}$ atoms produced by electron impact dissociation was determined as follows. The dissociation of molecular hydrogen by lowenergy electrons occurs mainly via a dissociative excitation process through the lowest repulsive $b^{3} \Sigma_{u}^{+}$state. The $\mathrm{H}(1 \mathrm{~s})$ atoms produced in this process have kinetic energy which is estimated to be $\sim 2-3 \mathrm{eV}$ [26]. These hot $\mathrm{H}$ atoms are cooled in collisions with Ar atoms. On average 85 collisions are needed to cool the hot $\mathrm{H}$ atoms to thermal energies. Using the collision frequency calculated with the cross section from [27] it was estimated that at our experimental conditions the 
hot $\mathrm{H}$ atoms are cooled down to the gas temperature within $8 \mathrm{~ns}$. So it was considered in the model that the recombining and diffusing $\mathrm{H}$ atoms have a temperature equal to the gas temperature. The gas temperature used in the model was $T_{\mathrm{g}}=300 \mathrm{~K}$, since most of the area investigated by the model lay outside the active discharge, where the gas temperature was close to the room temperature. It was also found that the results of the model with higher gas temperature (up to $500 \mathrm{~K}$ ) are not significantly different from the results calculated with $T_{\mathrm{g}}=300 \mathrm{~K}$. The values of the recombination coefficients were taken from [28]. The value of the diffusion coefficient $D=1.4 \mathrm{~cm}^{2} \mathrm{~s}^{-1}$ was taken from [29]. Equation (4) was solved numerically by the methods of lines for $x$ from 0 to $x_{\max }=1.2$ $\mathrm{cm}$. The boundary conditions were

$$
n\left(x_{\max }, t\right)=0
$$

and

$$
-D \frac{\partial n}{\partial x}=-\gamma \frac{1}{4} v_{\text {th }} n+Q(t) \quad \text { at } x=0,
$$

where $v_{\text {th }}$ is the mean thermal velocity of the $\mathrm{H}$ atoms; $\gamma$ is the surface recombination probability for the $\mathrm{H}$ atoms and $Q(t)$ is the source term, which describes a possible source of $\mathrm{H}$ atoms from the dielectric surface. The value of $\gamma$ at the temperature of $300 \mathrm{~K}$ was set to $10^{-4}$ in the model [30]. The experimental data on $\mathrm{H}$ atom concentration at $t=1 \mu \mathrm{s}$ were fitted by an exponential function. Afterwards the $\mathrm{H}$ atom concentrations calculated using this exponential function were taken as the initial values for the solution of equation (4).

The atomic hydrogen concentration calculated by the model without any surface source of $\mathrm{H}$ atoms is shown as solid lines in figures 6 and 7. With the exception of the points located closest to the surface of the dielectrics, the model agrees well with the temporal behaviour found by our TALIF measurements. The model predicts the existence of a concentration plateau followed by the fall of atomic hydrogen concentration. Both the duration of the plateau and the rate of concentration fall calculated by the model are in reasonable agreement with the experiment. For some points above the bottom electrode (positions $0.3 \mathrm{~mm}$ and $0.5 \mathrm{~mm}$ in figure 7) the calculated concentration values are shifted with respect to the measured data. This originates from the fact that the measured spatial concentration profile did not fit perfectly to the exponential fall function, used as the initial condition for the model.

According to the model the existence of the plateau in the temporal development of atomic hydrogen concentration can be explained as a mutual effect of recombination and diffusion. The recombination losses of hydrogen atoms in regions farther from the dielectric surface are for some time compensated by the diffusion of atomic hydrogen from regions close to the dielectric, where the concentration values are relatively high. The model does not predict the existence of a plateau at the closest vicinity to the surface since there is no diffusion supply of atomic hydrogen to this region. Nevertheless, our measurement revealed that a plateau is present even in the closest vicinity of the dielectric surface. This discrepancy between the model outputs and measurements indicates that the dielectric surface acts in the afterglow as a source of free hydrogen atoms that were either adsorbed on the surface or directly created on the surface e.g. by surface ion recombination. To prove this hypothesis the model was extended by a source of atomic hydrogen released from the dielectric surface. The flux $Q$ of $\mathrm{H}$ atoms from the surface was described by the following function:

$$
Q(t)=A \exp (-k t)
$$

The constants $A$ and $k$ in this function were chosen so as to achieve good agreement between the measurement and calculation data. The atomic hydrogen concentration calculated by the model with this atomic hydrogen flux is shown as dash-and-dot lines in figures 6 and 7. Good agreement of concentrations was obtained in front of the bottom electrode for $A=1 \times 10^{18} \mathrm{~cm}^{-2} \mathrm{~s}^{-1}$ and $k=1 \times 10^{5} \mathrm{~s}^{-1}$; see figure 7 . The flux term increased the hydrogen concentration for the three nearest distances from the dielectric surface at a time delay of around $10 \mu \mathrm{s}$, which gave significantly better agreement with the measurement data. The concentration of atomic hydrogen at positions more distant from the dielectric surface was not affected. In front of the inter-electrode gap, good agreement of the calculated concentration values with the experimental ones was obtained for $A=2.7 \times 10^{18} \mathrm{~cm}^{-2} \mathrm{~s}^{-1}$ and $k=1 \times 10^{5} \mathrm{~s}^{-1}$; see figure 6 . In this case there was higher flux of atomic hydrogen from the surface, which could be related to the higher $\mathrm{H}$ atom concentration in this region. It is clear that in both cases introduction of the flux term improved the numerical fit to our experimental data in the close vicinity of the dielectric surface.

If the $\mathrm{H}$ atom flux from the surface is due to desorption, then it follows from equation (9) that the first-order desorption rate coefficient $k_{\text {desor }}$ is equal to $k=1 \times 10^{5} \mathrm{~s}^{-1}$. This rate coefficient can be shown to have an Arrhenius form

$$
k_{\text {desor }}=k_{0} \exp \left(\frac{-E_{\text {desor }}}{k_{\mathrm{B}} T}\right),
$$

where $E_{\text {desor }}$ is the depth of the potential well and for chemisorption $k_{0} \sim 10^{13}-10^{15} \mathrm{~s}^{-1}$ [31]. If $k_{0}$ is set to $10^{14} \mathrm{~s}^{-1}$, then the value $E_{\text {desor }}=0.5 \mathrm{eV}$ is obtained. Also the constant $A$ is equal to $n_{\mathrm{S} 0} k_{\text {desor, }}$, where $n_{\mathrm{S} 0}$ is the initial surface concentration of absorbed $\mathrm{H}$ atoms. For the position in front of the bottom electrode and inter-electrode gap the values of $n_{\mathrm{S} 0}$ are $1 \times 10^{13} \mathrm{~cm}^{-2}$ and $2.7 \times 10^{13} \mathrm{~cm}^{-2}$, respectively. Since no other studies dealing with $\mathrm{H}$ atom desorption from a quartz surface were found, the obtained values of $n_{\mathrm{S} 0}$ and $E_{\mathrm{des} o r}$ were compared with values published in studies of the desorption of other atoms: Marinov et al [32] estimated the initial surface concentration of $\mathrm{N}$ atoms on a Pyrex surface to be $3 \times 10^{13}$ $\mathrm{cm}^{-2}$, and Guaitella et al [33] estimated the initial surface concentration of $\mathrm{O}$ atoms on a Pyrex surface to be $2 \times 10^{14}$ $\mathrm{cm}^{-2}$. Marinov et al [34] also studied the kinetics of adsorption, desorption and recombination of nitrogen atoms on a silica surface and they concluded that weakly bonding active sites exist on the surface with the binding energy smaller than $1 \mathrm{eV}$. To conclude, the obtained values of $n_{\mathrm{S} 0}$ and $E_{\text {desor }}$ for $\mathrm{H}$ atom desorption from quartz surfaces are consistent with the abovementioned references. 


\section{Conclusion}

The difficulties of employing TALIF for the measurement of atomic hydrogen in the vicinity of the dielectric surface of coplanar DBD can be solved by a suitable choice of dielectric material with a smooth surface and low intrinsic fluorescence; by the proposed treatment of redundant signals; by taking measurements during the afterglow phase, so no artificial laser breakdown could be ignited; and by employing coplanar DBD with a convexly curved surface.

The spatio-temporal profile of atomic hydrogen concentration shows that free hydrogen atoms are localized mainly in the $0.3 \mathrm{~mm}$ thin layer above the dielectric surface. This corresponds well to the thickness of the active discharge measured by discharge imaging. The area with the highest concentration of atomic hydrogen is situated in the closest vicinity of the dielectric surface and its value is above $10^{22} \mathrm{~m}^{-3}$. The lateral diffusion of atomic hydrogen from the axis of localized filaments is approx. $1 \mathrm{~mm}$. The atomic hydrogen concentration measured in the coplanar surface DBD is significantly higher than the values reported for volume DBD.

The temporal evolution of atomic hydrogen concentration shows that the atomic hydrogen concentration stays constant for a certain time after the end of the laser pulse. This concentration plateau lasts from tens of microseconds for positions close to the solid surface, to approximately $1 \mathrm{~ms}$ for positions located $1 \mathrm{~mm}$ above the surface. Our 1D numerical model explains the formation of the plateau by the combination of the recombination and diffusion of hydrogen atoms. The recombination losses of atomic hydrogen in regions farther away from the dielectric are compensated by the diffusion flow from $\mathrm{H}$-atom-rich regions that are located closer to the dielectric. The fact that the plateau remains for a relatively long time even at the closest vicinity of the dielectric surface indicates that the dielectric surface itself acts for tens of microseconds as a source of atomic hydrogen. The spatio-temporal development of atomic hydrogen is very similar in both investigated regions, i.e. in the center of the inter-electrode gap and above the HV electrode.

\section{Acknowledgments}

This research has been supported by the Czech Science Foundation under contract GA13-24635S, by the project CZ.1.05/2.1.00/03.0086 funded by the European Regional Development Fund, and by projects LO1411 (NPU I) and 7AMB14SK204 funded by the Ministry of Education, Youth and Sports of the Czech Republic.

\section{References}

[1] Chang R P H, Chang C C and Darack S 1982 J. Vac. Sci. Technol. 2045
[2] Pearton S J, Corbett J W and Shi T S 1987 Appl. Phys. A 43153

[3] Korner N, Beck E, Dommann A, Onda N and Ramm J 1995 Surf. Coat. Technol. 76-7 731

[4] Kromka A, Čech J, Kozak H, Artemenko A, Ižák T, Čermák J, Rezek B and Černák M 2015 Phys. Status Solidi b 2522602

[5] Schröder V, Emonts B, Janßen H and Schulze H-P 2004 Chem. Eng. Technol. 27847

[6] Shebeko Yu N, Tsarichenko S G, Trunev A V, Korolchenko A Ya and Kaplin A Yu 1994 Combust. Explosion Shock Waves 30183

[7] Bogaerts A and Gijbels R 2002 Spectrochim. Acta B 571071

[8] Jiménez-Redondo M, Cueto M, Doménech J L, Tanarro I and Herrero V J 2014 RSC Adv. 462030

[9] Amorim J, Baravian G and Jolly J 2000 J. Phys. D: Appl. Phys. 33 R51

[10] Niemi K, Schulz-von der Gathen V and Döbele H F 2005 Plasma Sources Sci. Technol. 14375

[11] Döbele H F, Mosbach T, Niemi K and Schulz-von der Gathen V 2005 Plasma Sources Sci. Technol. 14 S31

[12] van Gessel A F H, van Grootel S C and Bruggeman P J 2013 Plasma Sources Sci. Technol. 22055010

[13] Bruggeman P and Brandenburg R 2013 J. Phys. D: Appl. Phys. 46464001

[14] Ono R 2016 J. Phys. D: Appl. Phys. 49083001

[15] Boogaarts M G H, Mazouffre S, Brinkman G J, van der Heijden H W P, Vankan P, van der Mullen J A M, Schram D C and Döbele H F 2002 Rev. Sci. Instrum. 7373

[16] Niemi K, Schulz-von der Gathen V and Döbele H F 2001 J. Phys. D: Appl. Phys. 342330

[17] Bittner J, Kohse-Höinghaus K, Meier U and Just T 1988 Chem. Phys. Lett. 143571

[18] Ambrico P F, Ambrico M, Šimek M, Colaianni A, Dilecce G and De Benedictis S 2009 Appl. Phys. Lett. 94231501

[19] Voráč J, Dvořák P, Procházka V, Morávek T and Ráhel J 2015 Eur. Phys. J. Appl. Phys. 7120812

[20] Es-Sebbar E T, Sarra-Bournet Ch, Naudé N, Massines F and Gherardi N 2009 J. Appl. Phys. 106073302

[21] Jolly J and Booth J P 2005 J. Appl. Phys. 97103305

[22] Dvořák P, Talába M, Obrusnik A, Kratzer J and Dědina J 2016 in preparation

[23] Chérigier L, Czarnetzki U, Luggenhölscher D and Schulz-von der Gathen V 1999 J. Appl. Phys. 85696

[24] Lavrov B P, Lang N, Pipa A V and Röpcke J 2006 Plasma Sources Sci. Technol. 15147

[25] Šimor M, Ráhel J, Vojtek P, Černák M and Brablec A 2002 Appl. Phys. Lett. 812716

[26] Tawara H, Itikawa Y, Nishimura H and Yoshino M 1990 J. Phys. Chem. Ref. Data 19617

[27] Phelps A V 1992 J. Phys. Chem. Ref. Data 21883

[28] Baulch D L et al 1994 J. Phys. Chem. Ref. Data 23847

[29] Blyth G, Clifford A A, Gray P and Waddicor J I 1987 J. Chem. Soc. Faraday Trans. I 83751

[30] Kim Y C and Boudart M 1991 Langmuir 72999

[31] Zangwill A 1988 Physics at Surfaces (Cambridge: Cambridge University Press)

[32] Marinov D, Guaitella O, Rousseau A and Ionikh Y 2010 J. Phys. D: Appl. Phys. 43115203

[33] Guaitella O, Hübner M, Welzel S, Marinov D, Röpcke J and Rousseau A 2010 Plasma Sources Sci. Technol. 19045026

[34] Marinov D, Guaitella O, de los Arcos T, von Keudell A and Rousseau A 2014 J. Phys. D: Appl. Phys. 47475204 